\title{
温度场下多跨输流管道共振可靠性 及变量重要性测度分析
}

\author{
吴楠，郭庆，全国军，刘永寿
}

(西北工业大学 力学与土木建筑学院, 陕西 西安 710072)

\begin{abstract}
摘 要: 输流管道面临宽频激励导致的复杂多阶共振失效问题。为解决该问题,采用动刚度法对考虑 温度效应的多跨输流管道动力学方程进行了求解, 基于得到的固有频率, 结合共振失效规律建立了多 阶防共振系统可靠性分析模型。为分析输入变量不确定性对系统共振失效概率的影响程度,进一步 建立了输入变量方差重要性测度指标, 并通过引入主动学习 Kriging (ALK) 模型实现了共振失效概率 和重要性测度指标的高效求解。通过算例, 分析了液体流速、压强和温度效应对输流管道共振失效概 率的影响规律, 结果对于输流管道防共振优化设计具有重要指导意义。
\end{abstract}

\section{关 键 词: 输流管道; 动刚度法; 共振可靠性; 重要性测度; ALK}

中图分类号:TG156

文献标志码: A

管道作为气液输运与储存的重要载体, 在航空 航天、能源、生物等领域得到了广泛应用。在设计、 制造、组装、服役等全寿命周期内,管道结构参数的 不确定性大量存在, 进而导致管道固有频率的不确 定性。此外, 输流管道蕴含丰富的流固耦合作用, 会 进一步加剧管道固有频率的不确定性。而当固有频 率与激振力频率接近到一定程度会引发共振失效问 题。因此, 对输流管道开展防共振可靠性和重要性 测度分析是很必要的。

国内外关于工程结构面临的防共振失效问题开 展了大量的研究。Zhai 等 ${ }^{[1]}$ 采用精细响应面法对 输流管道开展了共振和动力可靠性分析, 并根据结 果对输流管道结构参数进行了优化。Guo 等 ${ }^{[2]}$ 对多 跨功能梯度输流管道进行了共振可靠性和全局灵敏 度分析, 并引人了主动学习 Kriging 模型提高计算效 率。张屹尚等 ${ }^{[3]}$ 采用 Kriging 模型分析了充液管道 非概率共振可靠性问题。韩涛等 ${ }^{[4]}$ 采用直曲组集 方法针对复杂液压管路实现了高效建模与模态分 析, 分析了管道布局对固有频率的影响。Ritto 等 ${ }^{[5]}$ 建立了一种考虑建模误差的流固耦合概率模型, 并
文章编号: 1000-2758(2021) 05-1035-08

将其应用于输流管道振动分析, 通过随机特征值分 析得到了相关颤振现象。

为研究随机变量对系统共振失效概率的影响程 度, 开展变量重要性测度分析是必要的。由于输流 管道涉及到多阶共振失效, 即需要考虑多输出响应, 需对多输出响应整体进行变量重要性测度分析。徐 立扬等 ${ }^{[6]}$ 提出了基于谱分解加权摩尔彭罗斯马氏 距离的多输出响应重要性测度方法。石岩等 ${ }^{[7]}$ 采 用包络函数法将时空动态可靠性问题转化为静态问 题, 实现了可靠性及重要性测度的高效分析。任超 和李洪双 ${ }^{[8]}$ 采用交叉熵方法提出了一种基于失效 概率的全局重要性测度分析方法。Wei 等 ${ }^{[9]}$ 提出了 一对模式重要性测度指标, 可有效分析各失效模式 对系统失效概率和可靠度的影响程度。

本文将动刚度法与 ALK 方法相结合, 采用前者 进行温度场下多跨输流管道固有频率的计算, 再采 用 ALK 方法计算不确定性参数影响下的多阶共振 失效概率, 并分别计算不同流速、压强和温度效应下 共振失效概率的大小, 分析不同工况对管道共振失 效概率的影响规律, 为输流管道的防共振设计提供 
参考。

\section{1 输流管道模态求解}

嵌人温度变化矩阵的多跨输流管道模型如图 1 所示。管道长度为 $L_{1}+L_{2}, U$ 表示流速, $R_{\mathrm{i}}$ 和 $R_{0}$ 分别 代表管道内径和外径。根据 Euler-Bernoulli 梁理 论, 内嵌温度变化矩阵的多跨管道的振动控制方 程为 ${ }^{[10]}$

$$
\frac{\partial Q}{\partial x}=m_{\mathrm{p}} \frac{\partial^{2} w}{\partial t^{2}}+K_{W} w-\frac{\partial}{\partial x}\left(N_{x} \frac{\partial w}{\partial x}\right)+F_{w}
$$

式中: $w$ 是管道的横向位移; $m_{\mathrm{p}}$ 是单位长度管道质 量; $N_{x}$ 是管道轴向力; $t$ 是时间。

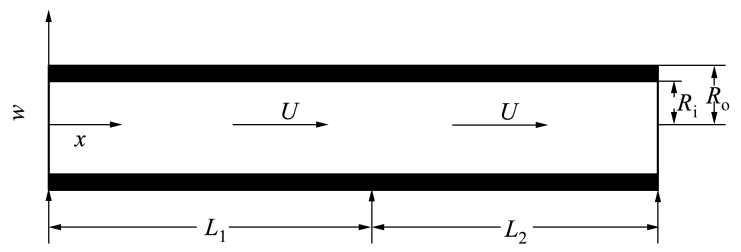

图 1 嵌人温度变化矩阵多跨输流管道模型

$$
\begin{aligned}
& Q=\frac{\partial M}{\partial x} \\
& M=-(E I) \frac{\partial^{2} w}{\partial x^{2}} \\
& K_{W}=K_{W 0}-a \Delta T
\end{aligned}
$$

式中: $Q, M$ 和 $K_{W}$ 分别是剪切力, 弯矩和温度变化刚 度; $(E I)$ 是弯曲刚度; $K_{W 0}$ 是初始温度变化刚度; $a$ 是温度变化系数; $\Delta T$ 表示温度变化值。

$F_{w}$ 由离心力, 科氏力和惯性力组成, 即

$$
F_{w}=M_{\mathrm{f}}\left(U^{2} \frac{\partial^{2} w}{\partial x^{2}}+2 U \frac{\partial^{2} w}{\partial x \partial t}+\frac{\partial^{2} w}{\partial t^{2}}\right)
$$

式中, $M_{\mathrm{f}}$ 是管道中单位长度流体的质量。

$$
\begin{aligned}
& N_{x}=N_{T}+N_{m} \\
& N_{m}=-p A_{f} \\
& N_{T}=-\frac{E A a}{1-2 \nu} \Delta T
\end{aligned}
$$

式中: $N_{m}$ 是由内部流体压强造成的附加轴向力; $N_{T}$ 是由温度变化导致的轴向力; $p$ 和 $A_{\mathrm{f}}$ 分别是流体压 强和流体截面积。

将方程 (2) (6) 代人方程 (1), 振动控制方程 可重写为

$$
(E I) * \frac{\partial^{4} w}{\partial x^{4}}+\left(M_{\mathrm{f}} U^{2}+p A_{\mathrm{f}}-N_{x}\right) \frac{\partial^{2} w}{\partial x^{2}}+
$$

$$
2 M_{\mathrm{f}} U \frac{\partial^{2} w}{\partial x \partial t}+\left(M_{\mathrm{f}}+m_{\mathrm{p}}^{*}\right) \frac{\partial^{2} w}{\partial t^{2}}+K_{W} w=0
$$

式中, $(E I)^{*}$ 和 $m_{\mathrm{p}}^{*}$ 分别表示有效抗弯刚度和有效 单位长度管道质量。

采用动刚度法 ${ }^{[11]}$ 求解管道固有频率。假设运 动微分方程的通解为

$$
w(x, t)=W(x) \mathrm{e}^{\mathrm{i} \omega t}
$$

式中: $W(x)$ 表示横向位移在频域内的设解; $\omega$ 表示 固有圆频率; $\mathrm{i}$ 为虚数单位。

将通解代人方程 (7), 得到频域内的振动方程

$$
\begin{aligned}
& (E I) * \frac{\partial^{4} W}{\partial x^{4}}+\left(M_{\mathrm{f}} U^{2}+p A_{\mathrm{f}}-N_{x}\right) \frac{\partial^{2} W}{\partial x^{2}}+ \\
& 2 \mathrm{i} \omega M_{\mathrm{f}} U \frac{\partial W}{\partial x}+\left(K_{W}-\omega^{2}\left(M_{\mathrm{f}}+m_{\mathrm{p}}^{*}\right)\right) W=0
\end{aligned}
$$

进一步地将位移在频域内的解设为

$$
W(x)=c \mathrm{e}^{\mathrm{i} k x}
$$

式中: $c$ 表示待定常数; $k$ 表示波数。

将方程 (10) 代人散射方程 (9) 得到

$$
\begin{gathered}
(E I)^{*}(1+\mathrm{i} \omega \alpha) k^{4}-\left(M_{\mathrm{f}} U^{2}+p A_{\mathrm{f}}\right) k^{2}- \\
2 \omega M_{\mathrm{f}} U k-\omega^{2}\left(M_{\mathrm{f}}+m_{\mathrm{p}}^{*}\right)=0
\end{gathered}
$$

式中, $\alpha$ 表示结构内阻损耗因子。

根据以上方程, 可知波数 $k$ 有 4 个解, 则位移在 频域内的解可以设为

$$
W(\omega, x)=\sum_{j=1}^{4} w_{j} \mathrm{e}^{\mathrm{i} k_{j} x}
$$

管道的转角、弯矩和剪力在频域内的解为

$$
\begin{aligned}
& \phi(\omega, x)=\sum_{j=1}^{4} \mathrm{i} k_{j} w_{j} \mathrm{e}^{\mathrm{i} k_{j} x} \\
& M(\omega, x)=\sum_{j=1}^{4}-k_{j}^{2}(E I)^{*} w_{j} \mathrm{e}^{\mathrm{i} k_{j} x} \\
& Q(\omega, x)=\sum_{j=1}^{4} \mathrm{i} k_{j}^{3}(E I)^{*} w_{j} \mathrm{e}^{\mathrm{i} k_{j} x}
\end{aligned}
$$

多跨管道的第 $m$ 跨单元节点位移如图 2 所示。

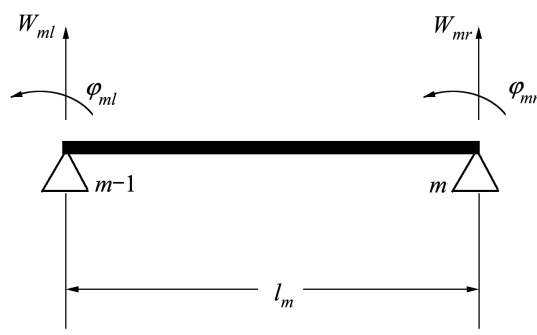

图 2 第 $m$ 跨单元示意图 


$$
\begin{aligned}
& W_{m \mathrm{l}}=W(0), \phi_{m \mathrm{l}}=W^{\prime}(0), \\
& W_{m \mathrm{r}}=W\left(l_{m}\right), \phi_{m \mathrm{r}}=W^{\prime}\left(l_{m}\right) \\
& M_{m \mathrm{l}}=-M(0), Q_{m \mathrm{l}}=-Q(0), \\
& M_{m \mathrm{r}}=M\left(l_{m}\right), Q_{m \mathrm{r}}=Q\left(l_{m}\right)
\end{aligned}
$$

式中: $l_{m}$ 表示第 $m$ 跨管道单元的长度;下标 $\mathrm{l}$ 和 $\mathrm{r}$ 分 别表示单元的左端和右端。

为了得到第 $m$ 跨单元的动刚度矩阵, 单元的节 点位移在局部坐标系里为

$\left\{\begin{array}{l}W_{m \mathrm{l}} \\ \phi_{m \mathrm{l}} \\ W_{m \mathrm{r}} \\ \phi_{m \mathrm{r}}\end{array}\right\}=\left[\begin{array}{cccc}1 & 1 & 1 & 1 \\ \lambda_{1} & \lambda_{2} & \lambda_{3} & \lambda_{4} \\ \mathrm{e}^{\mathrm{i} k_{1} l_{m}} & \mathrm{e}^{\mathrm{i} k_{2} l_{m}} & \mathrm{e}^{\mathrm{i} k_{3} l_{m}} & \mathrm{e}^{\mathrm{i} k_{4} l_{m}} \\ \lambda_{1} \mathrm{e}^{\mathrm{i} k_{1} l_{m}} & \lambda_{2} \mathrm{e}^{\mathrm{i} k_{2} l_{m}} & \lambda_{3} \mathrm{e}^{\mathrm{i} k_{3} l_{m}} & \lambda_{4} \mathrm{e}^{\mathrm{i} k_{4} l_{m}}\end{array}\right] \cdot\left\{\begin{array}{l}w_{1} \\ w_{2} \\ w_{3} \\ w_{4}\end{array}\right\}$

式中, $\lambda_{j}=i k_{j}(j=1,2,3,4)$ 。

将(18) 式写成矩阵向量形式为

$$
\boldsymbol{W}_{m}=\boldsymbol{Y}_{m}(\omega) \boldsymbol{w}_{m}
$$

式中, $\boldsymbol{W}_{m}, \boldsymbol{Y}_{m}$ 和 $\boldsymbol{w}_{m}$ 分别表示位移向量、系数矩阵和 系数向量。

那么,第 $m$ 跨单元的节点力向量为

$$
\left\{\begin{array}{c}
-Q_{m l} \\
-M_{m l} \\
Q_{m \mathrm{r}} \\
M_{m \mathrm{r}}
\end{array}\right\}=\left[\begin{array}{cccc}
-\gamma_{1} & -\gamma_{2} & -\gamma_{3} & -\gamma_{4} \\
-\beta_{1} & -\beta_{2} & -\beta_{3} & -\beta_{4} \\
\gamma_{1} \mathrm{e}^{\mathrm{i} k_{1} l_{m}} & \gamma_{2} \mathrm{e}^{\mathrm{i} k_{2} l_{m}} & \gamma_{3} \mathrm{e}^{\mathrm{i} k_{3} l_{m}} & \gamma_{4} \mathrm{e}^{\mathrm{i} k_{4} l_{m}} \\
\beta_{1} \mathrm{e}^{\mathrm{i} k_{1} l_{m}} & \beta_{2} \mathrm{e}^{\mathrm{i} k_{2} l_{m}} & \beta_{3} \mathrm{e}^{\mathrm{i} k_{3} l_{m}} & \beta_{4} \mathrm{e}^{\mathrm{i} k_{4} l_{m}}
\end{array}\right] \cdot\left\{\begin{array}{l}
w_{1} \\
w_{2} \\
w_{3} \\
w_{4}
\end{array}\right\}
$$

式中, $\gamma_{j}=\mathrm{i} k_{j}^{3}(E I)^{*}, \beta_{j}=-k_{j}^{2}(E I)^{*}, j=1,2,3,4$ 。

相应地, (20) 式的矩阵向量形式为

$$
\boldsymbol{F}_{m}=\boldsymbol{\Pi}_{m}(\omega) \boldsymbol{w}_{m}
$$

式中: $\boldsymbol{\Pi}_{m}(\omega)$ 表示系数矩阵; $\boldsymbol{F}_{m}$ 表示节点力矩阵。

则第 $m$ 跨单元的节点力向量和节点位移向量 间的关系为

$$
\boldsymbol{F}_{m}=\boldsymbol{K}_{m}(\omega) \boldsymbol{w}_{m}
$$

式中: $\boldsymbol{K}_{m}$ 表示第 $m$ 跨单元的动刚度矩阵,

$\boldsymbol{K}_{m}(\omega)=\boldsymbol{\Pi}_{m}(\omega) \boldsymbol{Y}_{m}(\omega)^{-1}$ 。

根据以上方法可以建立其他各跨的动刚度矩 阵, 进而参考有限元法进行单元动刚度矩阵组装, 构 建多跨输流管道在全局坐标系下的节点位移和节点 力之间的关系为

$$
\boldsymbol{K}_{g}(\omega) \boldsymbol{W}_{g}=\boldsymbol{F}_{g}
$$

式中, $K_{g}, W_{g}$ 和 $F_{g}$ 分别表示全局坐标系下的动刚度 矩阵、位移向量和节点力向量。

方程 (23) 取得非零解的充要条件为系数矩阵
行列式为零, 即

$$
h(\omega)=\operatorname{det}\left[\boldsymbol{K}_{c g}\right]=0
$$

通过 (24) 式可以求出多跨输流管道的固有频率。

\section{2 共振可靠性与重要性分析}

假设结构固有频率为 $R$, 激振力频率为 $S$,一般 认为当固有频率和激振力频率接近到一定程度时, 结构会因共振而造成破坏。首先考虑一阶共振失效 的情况, 用 $Z_{1}$ 来表示固有频率和激振力频率的接近 程度, 则 $Z_{1}$ 的表达式为

$$
Z_{1}(\boldsymbol{X})=\frac{\left|R_{1}-S\right|}{R_{1}}
$$

式中: $R_{1}$ 表示一阶固有频率; $\boldsymbol{X}$ 表示随机输人变量; $\boldsymbol{X}=\left\{x_{1}, x_{2}, \cdots, x_{n}\right\}$ ( $n$ 为变量维数)。

假设发生共振失效的阈值为 $q$, 那么一阶共振 可靠性的功能函数 $g_{1}(\boldsymbol{X})$ 为

$$
g_{1}(\boldsymbol{X})=Z_{1}(\boldsymbol{X})-q
$$

进一步考虑激振力频率的宽频特性, 可能导致 多阶固有频率发生共振, 将 $g_{1}$ 进行推广。假设第 $j j$ 阶共振可靠性的功能函数 $g_{j j}(\boldsymbol{X})$ 为

$$
g_{i j}(\boldsymbol{X})=Z_{j j}(\boldsymbol{X})-q
$$

式中, $Z_{i j}(X)=\frac{\left|R_{j j}-S\right|}{R_{i j}}, R_{j j}$ 为第 $j j$ 阶固有频率。

易知, 任意一阶固有频率发生共振失效均代表 结构整体失效, 即 $g_{i j}$ 之间是逻辑 “或” 的关系, 因此 建立多阶共振失效下的系统功能函数 $g(\boldsymbol{X})$ 为

$$
\left\{\begin{array}{lll}
g(\boldsymbol{X})<0 & \text { any } & g_{j j}<0 \\
g(\boldsymbol{X}) \geqslant 0 & \text { all } & g_{j j} \geqslant 0
\end{array}\right.
$$

共振失效概率 $P_{f}$ 为

$$
P_{f}=P\left\{\min _{v=1}^{i j} g_{v}(X)<0\right\}
$$

基于可靠性的变量重要性分析 (variable importance analysis, VIA) 方法旨在量化随机输人变量的 不确定性对失效概率的贡献, 对于单一失效模式的 结构构件, 该方法得到了广泛研究。本文将这些指 标推广到具有多种失效模式的结构体系。基于文献 [13], 可以将 Sobol 指数衍生出的 2 个 VIA 指标, 即 单个输人变量 $x_{v}$ 的主效应指标和总效应指标分别 定义为

$$
S_{v}=\frac{\operatorname{var}\left[\mathrm{E}\left(I_{F} \mid x_{v}\right)\right]}{\operatorname{var}\left(I_{F}\right)}
$$




$$
\begin{aligned}
S_{T_{v}} & =\frac{\operatorname{var}\left(I_{F}\right)-\operatorname{var}\left[\mathrm{E}\left(I_{F} \mid x_{\sim v}\right)\right]}{\operatorname{var}\left(I_{F}\right)}= \\
& \frac{\mathrm{E}\left[\operatorname{var}\left(I_{F} \mid x_{\sim v}\right)\right]}{\operatorname{var}\left(I_{F}\right)}
\end{aligned}
$$

式中: $x_{\sim v}$ 指包含所有除了 $x_{v}$ 的输人变量的向量; $\operatorname{var}(\cdot)$ 和 $\mathrm{E}(\cdot)$ 是方差和期望的数学运算符号。和 Sobol 的指标相似, 以可靠性为基础的 VIA 指标满足 $0 \leqslant S_{v} \leqslant S_{T v} \leqslant 1$ 。

\section{3 求解策略}

\section{1 主动学习 Kriging 模型}

Kriging 模型由两部分组成, 前半部分为全局近 似, 后半部分为局部偏差 ${ }^{[14]}$, 具体形式如下所示

$$
G(x)=F(x, \beta)+z(x)=f(x) \beta+z(x)
$$

式中: $G(x)$ 表示待拟合的目标响应函数; $f(x)$ 表示 变量 $x$ 的多项式; $\beta$ 表示 $f(x)$ 的系数; $z(x)$ 表示待拟 合函数的随机分布部分。

相应的预测值 $\hat{G}(x)$ 关于预测点 $x$ 的期望与方 差为

$$
\begin{gathered}
\mu_{G}(x)=\hat{\beta}+\boldsymbol{r}(\theta, x)^{\mathrm{T}} \boldsymbol{R}(\theta)^{-1}(\boldsymbol{g}-\hat{\beta} \boldsymbol{I}) \\
\sigma_{G}^{2}(x)=\sigma^{2}\left[\begin{array}{c}
\mathbf{1}+\frac{\left(\mathbf{1}^{\mathrm{T}} \boldsymbol{R}(\theta)^{-1} \boldsymbol{r}(\theta, x)-\mathbf{1}\right)^{2}}{\mathbf{1}^{\mathrm{T}} \boldsymbol{R}(\theta)^{-1} \mathbf{1}}- \\
\boldsymbol{r}(\theta, x)^{\mathrm{T}} \boldsymbol{R}(\theta)^{-1} \boldsymbol{r}(\theta, x)
\end{array}\right]
\end{gathered}
$$

式中: 1 是 $n$ 维单位列向量; $\boldsymbol{g}$ 是 $n$ 维列向量, 含有每 个设计点的目标响应值; $\boldsymbol{r}^{\mathrm{T}}(x)$ 是 $n$ 维列向量; $\boldsymbol{R}$ 表 示观测点 $x$ 与样本点 $\left\{x^{(1)}, x^{(2)}, \cdots, x^{(n)}\right\}$ 间的相 关性。

相关参数 $\theta$ 的值通过最大似然估计求解得到, 那么当相关函数是高斯相关函数时, 则可转化为下 面的优化问题

$$
\theta^{*}=\underset{\theta}{\operatorname{argmin}}\left(|\boldsymbol{R}|^{\frac{1}{n}} \hat{\sigma}^{2}\right)
$$

ALK 模型建立都在 Matlab 中的 DACE 工具箱 中完成, 相关参数 $\theta_{k}$ 的优化可以通过全局优化策略 的 DIRECT 优化算法 ${ }^{[15]}$ 得到。

目前较为常用的学习方程为

$$
U(x)=\frac{\left|\mu_{g}(x)\right|}{\sigma_{g}(x)}
$$

该 U 型函数由 Echard 等人 ${ }^{[16]}$ 于 2011 年在 AKMCS 方法中提出。

\subsection{ALK 方法的基本步骤}

1) 在不确定性域中随机抽取 $\boldsymbol{X}_{\Gamma}=\left(x_{1, \Gamma}, x_{2, \Gamma}\right.$, $\left.\cdots, x_{n, \Gamma}\right)(\Gamma=1,2, \cdots, N)$ 个初始样本点, 计算功能 函数值, 构建初始 Kriging 模型, 此处 $N=20$ 。

2) 随机产生大量候选样本点, 为 $U$ 型学习方程 选点做准备, 为使候选样本点充满不确定性域, 取候 选点数量为 $10^{5}$ 。

3) 在候选点中计算 Kriging 模型的预测值 $\mu_{G}(\boldsymbol{X})$ 和 $U$ 值, 将 $U$ 值最小的点记为 $\boldsymbol{X}^{*}$ 。

4) 若 $U$ 函数最小值满足收玫条件的阈值, 则转 人第 6$)$ 步,本文 $U$ 函数阈值大小为 2 。

$5)$ 若 4) 中的收玫条件无法满足, 将 $\boldsymbol{X}^{*}$ 加人 $\mathrm{DoE}$ 中, 计算 $\boldsymbol{X}^{*}$ 处的功能函数值并更新 Kriging 模 型,返回到第 3) 步。

6) 基于已建立的 Kriging 模型，代人 Monte-Carlo 法中求解防共振失效概率。

\section{4 算例分析}

管长 $L_{1}+L_{2}=15 \mathrm{~m}$, 外径 $R_{0}$, 壁厚 $d$, 管道密度 $\rho_{\mathrm{p}}$, 弹性模量 $E$, 流体密度 $\rho_{\mathrm{f}}$, 泊松比 $v=0.3, K_{0}=1 \times$ $10^{6} \mathrm{~N} / \mathrm{m}, a=5000 \mathrm{~N} /(\mathrm{m} \times \mathrm{K})$ 。外界激振力频率为 $S_{1}, S_{2}$ 和 $S_{3}$ 。随机变量及其分布信息如表 1 所示。 本文中的共振失效阈值为 $q=0.05$ 。

\section{表 1 输入变量统计信息}

\begin{tabular}{cccc}
\hline 输人变量/单位 & 分布类型 & 均值 & 标准差 \\
\hline$E / \mathrm{GPa}$ & Normal & 206 & 10.3 \\
$\rho_{\mathrm{p}} /\left(\mathrm{kg} \cdot \mathrm{m}^{3}\right)^{-1}$ & Normal & 7930 & 396.5 \\
$\rho_{\mathrm{f}} /\left(\mathrm{kg} \cdot \mathrm{m}^{3}\right)^{-1}$ & Normal & 1000 & 50 \\
$R_{\mathrm{o}} / \mathrm{m}$ & Normal & 0.2 & 0.01 \\
$d / \mathrm{m}$ & Normal & 0.01 & $5 \times 10^{-4}$ \\
$S_{1} / \mathrm{Hz}$ & Normal & 130 & 6.5 \\
$S_{2} / \mathrm{Hz}$ & Normal & 330 & 16.5 \\
$S_{3} / \mathrm{Hz}$ & Normal & 530 & 26.5 \\
$U /\left(\mathrm{m} \cdot \mathrm{s}^{-1}\right)$ & Normal & 0 & 0 \\
& & 10 & 0.5 \\
& & 20 & 1.0 \\
$p / \mathrm{MPa}$ & Normal & 0 & 0 \\
& & 14 & 0.70 \\
& & 21 & 1.05 \\
$\Delta T / \mathrm{K}$ & Normal & 1 & 0.05 \\
& & 10 & 0.5 \\
& & 20 & 1.0 \\
\hline
\end{tabular}




\section{1 液体流速的影响}

当压强和温度均为 0 时, 随着流速增大, 前六阶 固有频率如表 2 所示。可以明显看出, 随着流速的 增大, 各阶固有频率均逐渐降低, 且第一阶固有频率 降低最快, 该结果与参考文献 [2] 中的结论及理论 解释吻合。该现象是由于流速的增长导致了管道刚 度降低,进而导致固有频率降低。

表 2 不同液体流速下输流管道前六阶固有频率

\begin{tabular}{cccccccc}
\hline $\mathrm{U} /$ & \multirow{2}{*}{ 一阶 } & 二阶 & 三阶 & 四阶 & 五阶 & 六阶 \\
\hline 0 & 55.97 & 81.29 & 200.91 & 253.64 & 345.92 & 449.64 \\
10 & 54.92 & 81.25 & 200.86 & 253.60 & 345.89 & 449.60 \\
20 & 54.78 & 81.13 & 200.73 & 253.49 & 345.80 & 449.48 \\
\hline
\end{tabular}

本文考虑外激励频率为 130,330 和 $530 \mathrm{~Hz}$, 考 虑外激励的不确定性及输流管道固有频率的不确定 性, 管道可能在第二、四、六阶发生共振失效。因此, 本文考虑三阶共振问题, 其功能函数为

$$
\left\{\begin{array}{l}
g_{1}(X)=Z_{2}(X)-q \\
g_{2}(X)=Z_{4}(X)-q \\
g_{3}(X)=Z_{6}(X)-q
\end{array}\right.
$$

且该系统为串联系统, 系统功能函数为

$$
g=\min \left(g_{1}, g_{2}, g_{3}\right)
$$

本文采用 Monte-Carlo (MC) 方法计算得到的结 果作为精确解, 以此验证 ALK 方法的计算精度和效 率。不同流速条件下共振失效概率和函数调用次数 如表 3 所示。

\section{表 3 不同液体流速下的失效概率}

\begin{tabular}{ccccc}
\hline 方法 & 工况 & $\begin{array}{c}U / \\
\left(\mathrm{m} \cdot \mathrm{s}^{-1}\right)\end{array}$ & $P_{\mathrm{f}}$ & $\begin{array}{c}\text { 函数调 } \\
\text { 用次数 }\end{array}$ \\
\hline \multirow{4}{*}{ Monte-Carlo } & 1 & 0 & 0.0563 & $3 \times 10^{6}$ \\
& 2 & 10 & 0.0534 & $3 \times 10^{6}$ \\
& 3 & 20 & 0.0515 & $3 \times 10^{6}$ \\
\hline \multirow{3}{*}{ ALK } & 1 & 0 & 0.0561 & $20+189$ \\
& 2 & 10 & 0.0535 & $20+177$ \\
& 3 & 20 & 0.0519 & $20+201$ \\
\hline
\end{tabular}

ALK 方法的函数调用次数结果中, 第一项为初 始样本量, 第二项为通过主动学习增加的样本量。 可以发现, 随着流速的增加, 共振失效概率逐渐减 小。这是因为随着流速增加, 各阶固有频率均在下 降, 即在远离激振力频率, 所以共振失效可能性降 低。此外,对比 MC 和 ALK 2 种方法的结果可以发 现, ALK 方法得到的失效概率误差很小, 但功能函
数的调用次数极大地降低。 $\mathrm{MC}$ 法需要调用 $3 \times 10^{6}$ 次功能函数, 而 ALK 仅需要调用二百多次, 计算效 率极大提高。

当 $U=10 \mathrm{~m} / \mathrm{s}$ 时, 采用 ALK 法计算得到的 VIA 指标如图 3 所示。可以发现, $S_{R_{\mathrm{o}}}$ 和 $S_{E}$ 明显大于其他 变量的主指标, 说明 $R_{\mathrm{o}}$ 和 $E$ 对系统共振失效概率的 影响程度最大, 而其他变量的主指标趋近于零, 因此 在管道防共振设计中可忽略其他变量的不确定性, 降低问题的维度,减小计算负担。此外,各变量的总 指标的主要排序为 $R_{\mathrm{o}}>E>\rho_{\mathrm{p}}$, 总指标排序的前两 位与主指标排序的前两位相同, 说明 $R_{\mathrm{o}}$ 和 $E$ 对系统 共振失效概率的影响最大。同时所有变量的总指标 值明显大于主指标值, 说明变量间的相互作用对系 统共振失效概率有显著影响。

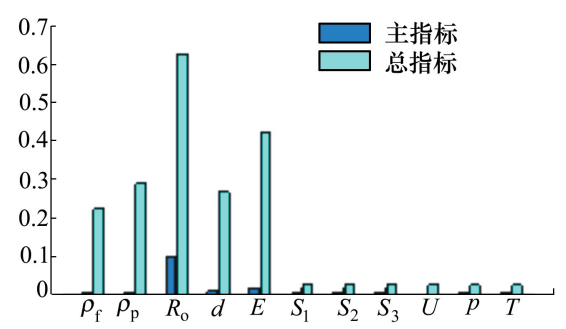

图 3 流速为 $10 \mathrm{~m} / \mathrm{s}$ 时的变量重要性测度指标

\section{2 液体压强的影响}

当 $U=0 \mathrm{~m} / \mathrm{s}, \Delta T=0 \mathrm{~K}$ 时,考虑压强的 3 种不同 工况, 即: (1) $p=0 \mathrm{MPa}$; (2) $p=14 \mathrm{MPa}$; (3p $p=28 \mathrm{MPa}_{\text {。 }}$ 随压强增大, 输流管道前六阶固有频率如表 4 所示。 可以发现，管道各阶固有频率均随压强增大而降低， 且下降趋势十分明显, 根据振动控制方程可知, 压强 $p$ 和流速 $U$ 对管道影响的作用类似。

表 4 不同液体压强下输流管道前六阶固有频率

\begin{tabular}{ccccccc}
\hline$p / \mathrm{MPa}$ & 一阶 & 二阶 & 三阶 & 四阶 & 五阶 & 六阶 \\
\hline 0 & 55.97 & 81.29 & 200.91 & 253.64 & 345.92 & 449.64 \\
14 & 46.77 & 75.05 & 192.42 & 246.39 & 340.46 & 441.22 \\
28 & 36.79 & 68.19 & 183.55 & 238.92 & 334.96 & 432.63 \\
\hline
\end{tabular}

3 种压强工况下的共振失效概率如表 5 所示。 由结果可知, 随着压强的增大, 系统共振失效概率不 断减小,其原因是压强的增大导致了固有频率的降 低, 使得固有频率逐渐远离激振力频率, 共振失效可 能性随之降低。ALK 法在保证计算精度的同时, 计 算效率明显优于 MC 法。 
表 5 不同液体压强下的失效概率

\begin{tabular}{ccccc}
\hline 方法 & 工况 & $p / \mathrm{MPa}$ & $P_{f}$ & $\begin{array}{c}\text { 函数调 } \\
\text { 用次数 }\end{array}$ \\
\hline \multirow{3}{*}{ Monte-Carlo } & 1 & 0 & 0.0563 & $3 \times 10^{6}$ \\
& 2 & 14 & 0.0312 & $3 \times 10^{6}$ \\
& 3 & 28 & 0.0180 & $3 \times 10^{6}$ \\
\hline \multirow{3}{*}{ ALK } & 1 & 0 & 0.0561 & $20+189$ \\
& 2 & 14 & 0.0312 & $20+178$ \\
& 3 & 28 & 0.0186 & $20+186$ \\
\hline
\end{tabular}

当 $p=14 \mathrm{MPa}$ 时,采用 ALK 法计算得到的 VIA 指标如图 4 所示。可以发现, $R_{\mathrm{o}}$ 和 $E$ 的主指标和总 指标均排在第一和第二位, 其中主指标明显大于其 他变量的主指标, 说明 $R_{\mathrm{o}}$ 和 $E$ 对系统共振失效概率 的影响程度最大, 而其他变量的主指标趋近于零, 因 此在管道防共振设计中可忽略其他变量的不确定 性。此外, 各变量的总指标的主要排序为 $R_{0}>E>$ $\rho_{\mathrm{p}}>\rho_{\mathrm{f}}$, 所有变量的总指标值明显大于主指标值, 其 规律与 $U=10 \mathrm{~m} / \mathrm{s}$ 时基本相同, 进一步说明了流速 $U$ 和液压 $p$ 相比其他随机变量, 对共振失效的影响 程度较小。

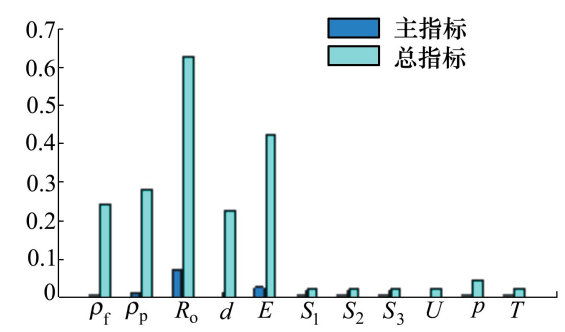

图 4 压强为 $14 \mathrm{MPa}$ 时的变量重要性测度指标

\section{3 温度效应的影响}

当 $U=0 \mathrm{~m} / \mathrm{s}, p=0 \mathrm{MPa}$ 时, 考虑温度效应的 3 种不同工况, 即: (1) $\Delta T=0 \mathrm{~K}$; (2) $\Delta T=10 \mathrm{~K}$; (3) $\Delta T=$ $20 \mathrm{~K}$ 。随温度变化值增大, 输流管道前六阶固有频 率如表 6 所示。可以发现, 管道各阶固有频率均随 温度变化值增大而降低,且下降趋势较为明显。

表 6 不同温度效应下输流管道前六阶固有频率

\begin{tabular}{ccccccc}
\hline$T / \mathrm{K}$ & 一阶 & 二阶 & 三阶 & 四阶 & 五阶 & 六阶 \\
\hline 0 & 55.97 & 81.29 & 200.91 & 253.64 & 345.92 & 449.64 \\
10 & 47.70 & 76.03 & 195.38 & 248.96 & 342.39 & 444.51 \\
20 & 39.09 & 70.36 & 189.70 & 244.18 & 338.84 & 439.31 \\
\hline
\end{tabular}

3 种温度效应工况下的共振失效概率如表 7 所 示。由结果可知, 随着温度变化值的增大, 系统共振
失效概率不断减小, 其原因与流速和压强的影响规 律相同。

表 7 不同温度效应下的失效概率

\begin{tabular}{ccccc}
\hline Method & Case & $T / \mathrm{K}$ & $P_{f}$ & $\begin{array}{c}\text { 函数调 } \\
\text { 用次数 }\end{array}$ \\
\hline \multirow{3}{*}{ Monte-Carlo } & 1 & 0 & 0.0563 & $3 \times 10^{6}$ \\
& 2 & 10 & 0.0409 & $3 \times 10^{6}$ \\
& 3 & 20 & 0.0296 & $3 \times 10^{6}$ \\
\hline \multirow{3}{*}{ ALK } & 1 & 0 & 0.0561 & $20+158$ \\
& 2 & 10 & 0.0404 & $20+164$ \\
& 3 & 20 & 0.0295 & $20+149$ \\
\hline
\end{tabular}

当 $\Delta T=20 \mathrm{~K}$ 时,采用 ALK 法计算得到的 VIA 指标如图 5 所示。可以发现, $R_{\mathrm{o}}$ 和 $E$ 的主指标和总 指标均排在第一和第二位, 其中主指标明显大于其 他变量的主指标, 而其他变量的主指标趋近于零, 各 变量的总指标的主要排序为 $R_{0}>E>t$, 同时所有变量 的总指标值明显大于主指标值, 主指标与总指标的 分布规律与 $U=10 \mathrm{~m} / \mathrm{s}$ 和 $p=14 \mathrm{MPa}$ 基本相同, 进 一步验证了 3 种变量对共振失效概率的影响程度较 小。VIA 指标对于管道的防共振设计具有重要参考 价值。

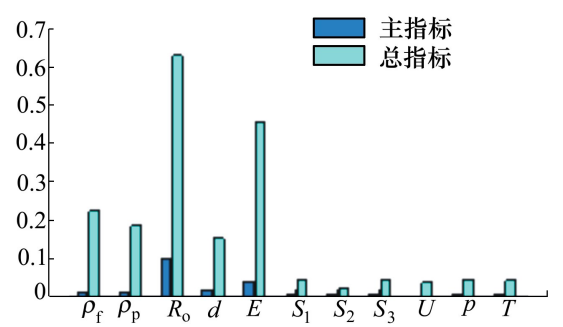

图 5 温度变化值为 $20 \mathrm{~K}$ 时的变量重要性测度指标

\section{5 结 论}

本文基于温度效应下的多跨输流管道模型建立 了共振可靠性和变量重要性分析方法, 并用算例分 析了流速、压强和温度效应对共振失效概率、VIA 的 影响。主要结论如下:

1) 流速、压强和温度变化值的增大均会导致管 道固有频率的降低, 造成固有频率与激振力频率逐 渐远离, 从而进一步导致系统共振失效概率的降低。

2) 各输人变量中, $R_{\mathrm{o}}$ 和 $E$ 对系统失效概率的 影响程度最大, 在设计中应控制或降低其不确定性 程度, 从而降低系统共振失效概率。

3) ALK 方法在保证计算精度的前提下, 极大地 


\section{提高了计算效率,有助于管道防共振可靠性分析方＼cjkstart法的工程应用。}

\section{参考文献:}

[1] ZHAI H B, WU Z Y, LIU Y S, YUE Z F. The dynamic reliability analysis of pipe conveying fluid based on a refined response surface method $[\mathrm{J}]$. Journal of Vibration and Control, 2015, 21(4) : 790-800

[2] GUO Q, LIU Y S, ZHAO Y Z, et al. Improved resonance reliability and global sensitivity analysis of multi-span pipes conveying fluid based on active learning Kriging model[J]. International Journal of Pressure Vessels and Piping, 2019, 170:92-101

[3] 张屹尚, 刘永寿, 赵涁, 等. 基于 Kriging 模型的充液管道共振可靠性分析 $[\mathrm{J}]$. 振动工程学报, 2012, 25(2) : 117-123 ZHANG Yishang, LIU Yongshou, ZHAO Bin, et al. Non-probabilistic reliability analysis on resonance of fluid-filled pipeline based on Kriging model $[\mathrm{J}]$. Journal of Vibration Engineering, 2014, 32(2) : 262-267 (in Chinese)

[4] 韩涛, 刘伟, 张子俊, 等. 基于直曲组集算法的复杂液压管路固有频率分析 $[\mathrm{J}]$. 振动与冲击, 2018, 37(7)：13-22 HAN Tao, LIU Wei, ZHANG Zijun, et al. Natural frequency analysis of complex hydraulic pipelines based on straight-curved pipeline assembly algorithm $[\mathrm{J}]$. Journal of Vibration and Shock, 2018, 37(7) : 13-22 (in Chinese)

[5] RITTO T G, SOIZE C, ROCHINHA F A, et al. Dynamic stability of a pipe conveying fluid with an uncertain computational model[J]. Journal of Fluids and Structures, 2014, 49: 412-426

[6] 徐立扬, 吕震宙, 王飞, 等. 多输出性能下的重要性测度指标及其求解方法 $[\mathrm{J}]$. 国防科技大学学报, 2017, 39(4): 154-160

XU Liyang, LYU Zhenzhou, WANG Fei, et al. Global sensitivity analysis for multiple outputs and their solutions [J]. Journal of National University of Defense Technology, 2017, 39(4): 154-160 (in Chinese)

[7] 石岩, 吕震宙, 周易成. 随时间和空间变化可靠性及可靠性全局灵敏度分析的包络函数法 $[\mathrm{J}]$. 西北工业大学学报, 2017, 35(4) : 591-598

SHI Yan, LYU Zhenzhou, ZHOU Yicheng. Temporal and spatial reliability and global sensitivity analysis with envelope functions [J]. Journal of Northwestern Polytechnical University, 2017, 35(4) : 591-598 (in Chinese)

～8 ］任超, 李洪双. 基于失效概率的全局重要性测度分析的交叉熵方法 $[\mathrm{J}]$. 西北工业大学学报, 2017, 35(3): 536-544

REN Chao, LI Hongshuang. Cross-entropy method for failure probability based global importance measure analysis $[\mathrm{J}]$. Journal of Northwestern Polytechnical University, 2017, 35(3): 536-544 (in Chinese)

[9] WEI P F, LIU F C, TANG C H. Reliability and reliability-based importance analysis of structural systems using multiple response Gaussian process model $[\mathrm{J}]$. Reliability Engineering and System Safety, 2018, 175: 183-195

[10] SADEGHI-GOYGHARI M, JEON S, KWON H J. Flutter instability of cantilevered carbon nanotubes caused by magnetic fluid flow subjected to a longitudinal field $[\mathrm{J}]$. Physica E: Low-dimensional System and Nanostructures, 2018, 98: 184-190

[11] KJOLSING E J, TODD M D. Frequency response of a fixed-fixed pipe immersed in viscous fluids, conveying internal steady flow [J]. Journal of Petroleum Science and Engineering, 2015, 134: 247-256

[12] DAI H L, RAO Y N, DAI T. A review of recent researches on FGM cylindrical structures under coupled physical interactions [J]. Composite Structures, 2016, 152: 199-225

[13] SALTELLI A. Making best use of model evaluations to compute sensitivity induces [J]. Computer Physics Communications, 2002, 145: 280-97

[14] GUO Q, LIU Y S, CHEN B Q, et al. A variable and mode sensitivity analysis method for structural system using a novel active learning Kriging model[J]. Reliability Engineering \& Structural Safety, 2021, 206: 107285

[15] LIU Q F, ZENG J P, YANG G. MrDIRECT: a multilevel robust direct algorithm for global optimization problems[J]. Journal of Global Optimization, 2015, $62(2): 205-227$

[16] ECHARD B, GAYTON N, LEMAIRE M. AK-MCS: an active learning reliability method combining Kriging and Monte Carlo simulation[J]. Structural Safety, 2011, 33:145-154 


\title{
Resonance reliability and importance measure analysis of multi-span pipe conveying fluid embedded in temperature-varying matrix
}

\author{
WU Nan, GUO Qing, TONG Guojun, LIU Yongshou
}

(School of Mechanics, Civil Engineering and Architecture, Northwestern Polytechnical University, Xi'an 710072, China)

\begin{abstract}
Pipe system conveying fluid faces the problem of multi-order resonance failure caused by broadband excitation. For solving above problem, the dynamic stiffness method is employed to solve the dynamic equations of multi-span pipes considering the temperature effect. Combining the obtained natural frequency and the rule of resonance failure of pipe system, a multi-order anti-resonance system reliability model is established in this paper. To analyze the effect of input variable uncertainty on the probability of system resonance failure, the variance-based importance measurement index is further established. By introducing the active learning Kriging (ALK) model, the resonance failure probability and importance measurement index can be calculated efficiently. The effects of fluid flow velocity, pressure and temperature on the probability of pipe resonance failure are analyzed in detail, which has significant guidance for the anti-resonance optimization design of pipes.
\end{abstract}

Keywords : pipe conveying fluid; dynamic stiffness method; resonance reliability; importance measure;ALK

引用格式: 吴楠, 郭庆, 全国军, 等. 温度场下多跨输流管道共振可靠性及变量重要性测度分析 $[J]$. 西北工业大学学报, 2021,39(5): 1035-1042

WU Nan, GUO Qing, TONG Guojun, et al. Resonance reliability and importance measure analysis of multi-span pipe conveying fluid embedded in temperature-varying matrix [J]. Journal of Northwestern Polytechnical University, 2021, 39(5): 1035-1042 (in Chinese) 